\section{First Reported Case of Shewanella haliotis in the Region of the Americas — New York, December 2018}

Dakai Liu, PhD ${ }^{1, *}$; Roberto Hurtado Fiel, $\mathrm{MD}^{2, *}$; Lucy Shuo Cheng, $\mathrm{MD}^{3, *}$; Takuya Ogami, $\mathrm{MD}^{2}$; Lulan Wang, $\mathrm{PhD}^{4}$; Vishnu Singh ${ }^{1}$; George David Rodriguez, PharmD ${ }^{5}$; Daniel Hagler, $\mathrm{MD}^{2}$; Chun-Chen Chen, MD, PhD ${ }^{2}$; William Harry Rodgers, MD, PhD ${ }^{1,6}$

On December 18, 2018, a man aged 87 years was evaluated in a hospital emergency department in Flushing, New York, for right lower abdominal quadrant pain. Evaluation included a computed tomography scan, which showed acute appendicitis with multiple abscesses measuring $\leq 3 \mathrm{~cm}$. The patient was admitted, a percutaneous drain was placed, and $5 \mathrm{~mL}$ of an opaque jelly-like substance was aspirated and sent for culture and testing for antimicrobial sensitivities.

Gram stain of the culture revealed gram-negative rods, and culture revealed monomicrobial 1-2-mm yellowish-brown mucoid colonies. ${ }^{\dagger}$ Sequencing of the isolate's $16 \mathrm{~S}$ ribosomal RNA revealed $>99.8 \%$ homology with Shewanella haliotis strain DW01 in the GenBank database. Antimicrobial susceptibility testing indicated that the isolate was susceptible to aminoglycosides, fluoroquinolones, certain penicillins, and broad-spectrum cephalosporins (Table). Biochemical tests were performed to characterize isolate (Supplementary Table, https://stacks.cdc.gov/view/cdc/83522). Phylogenetic analysis indicates that $S$. haliotis strain DW01 is the most recent ancestor of this clinical isolate. This is the first documented case of a $S$. haliotis appendix infection.

$S$. haliotis is an emerging human pathogen, first isolated from abalone gut microflora in 2007 (1). The geographic distribution of human infections caused by $S$. haliotis is concentrated in Asia, with most reports coming from China, Japan, South Korea, and Thailand (2). No cases of $S$. haliotis human infections had been reported in the World Health Organization's Region of the Americas.

The patient was treated empirically with intravenous piperacillin-tazobactam while in the hospital and was discharged with a prescription for oral amoxicillin-clavulanic acid. At a follow-up visit 13 days later, he was recovering well. Empiric treatment of Shewanella spp. can be challenging; limited and varying antibiotic susceptibility profiles have been reported

\footnotetext{
*These authors contributed equally to the report.

$\dagger$ After inoculation of specimen on sheep blood agar, chocolate agar, and MacKonkey agar plates and incubation at $98.6^{\circ} \mathrm{F}\left(37^{\circ} \mathrm{C}\right)$, beta-hemolysis was observed on the blood agar plates.
}

$(2,3)$. This patient's isolate was susceptible to several classes of antimicrobials, but resistance to certain antibiotics has been observed in this isolate and others (2). In a case series of 16 patients from Martinique, Shewanella spp. sensitivities to piperacillin-tazobactam and amoxicillin-clavulanic acid were reported to be $98 \%$ and $75 \%$, respectively (3).

Risk factors for or potential vectors of Shewanella spp. infections are unidentified in up to $40 \%-50 \%$ of cases (4). S. haliotis is ecologically distributed in marine environments, including broad contamination of cultivated shellfish. Although infection following consumption of seafood is seldom reported (5), consumption of raw seafood could be an important vehicle for foodborne illnesses and outbreaks. This patient reported consuming raw salmon 10 days before becoming ill but had no other marine exposures or exposure to ill contacts. The time from potential exposure to onset of abdominal pain in this patient is consistent with that reported in the literature on Shewanella spp. (3-49 days). The epidemiologic exposure history supports the link between raw fish consumption and infection.

No other organisms were isolated in this patient; in the Martinique case series of Shewanella spp., one half of infections were monomicrobial as well (3). This case highlights the importance of preventing seafood-associated infections and the need to consider rare human pathogens in elderly or immunocompromised, marine-exposed populations, as well as persons who might consume at-risk food that might have been imported from outside the United States and persons who might have been infected outside the United States when traveling.

\section{Acknowledgments}

Jun Young Choi, Debra Harragan Jokisch, Eric Konadu, Marian Memmer, Joseph Pilz, Giuseppe Pipia.

Corresponding authors: William Harry Rodgers, whr9001@nyp.org, 718-6701141; Chun-Chen Chen, chc4003@med.cornell.edu, 347-548-8777.

\footnotetext{
${ }^{1}$ Department of Pathology and Clinical Laboratories, NewYork-Presbyterian Queens, Flushing, New York; ${ }^{2}$ NewYork-Presbyterian Queens, Department of Surgery, Flushing, New York; ${ }^{3}$ Department of Dermatology, University of Pittsburgh Medical Center, Pittsburgh, Pennsylvania; ${ }^{4}$ Molecular Biology Institute, University of California, Los Angeles, California; ${ }^{5}$ Division of Infectious Diseases and Antimicrobial Stewardship, NewYork-Presbyterian Queens, Flushing, New York; ${ }^{6}$ Department of Pathology, Weill Cornell Medical College, New York City, New York.
}

All authors have completed and submitted the International Committee of Medical Journal Editors form for disclosure of potential conflicts of interest. No potential conflicts of interest were disclosed. 
TABLE. Antimicrobial sensitivity* of an isolate of Shewanella haliotis from an intraabdominal abscess - New York, December 2018

\begin{tabular}{|c|c|c|c|c|c|c|}
\hline \multirow[b]{2}{*}{ Antimicrobial } & \multirow[b]{2}{*}{ Drug class } & \multirow[b]{2}{*}{$\operatorname{MIC}(\mu \mathrm{g} / \mathrm{ml})$} & \multicolumn{3}{|c|}{ Range } & \multirow[b]{2}{*}{ Interpretation } \\
\hline & & & s & I & $\mathbf{R}$ & \\
\hline Amikacin & Aminoglycoside & 3 & $\leq 16$ & 32 & $\geq 64$ & $\mathrm{~s}$ \\
\hline Ampicillin & Penicillin & $\leq 4$ & $\leq 8$ & 16 & $\geq 32$ & $\mathrm{~S}$ \\
\hline Ampicillin-Sulbactam & Penicillin-beta-lactamase inhibitor & $>16 / 8$ & $\leq 8 / 4$ & $16 / 8$ & $\geq 32 / 16$ & I \\
\hline Aztreonam & Monobactam & $>16$ & $\leq 4$ & 8 & $\geq 16$ & $\mathrm{R}$ \\
\hline Cefazolin & Cephalosporin & $>16$ & $\leq 2$ & 4 & $\geq 8$ & $\mathrm{R}$ \\
\hline Cefepime & Cephalosporin & 0.094 & $\leq 2$ & $4-8$ & $\geq 16$ & $\mathrm{~S}$ \\
\hline Cefoxitin & Cephamycin & $>16$ & $\leq 8$ & 16 & $\geq 32$ & I \\
\hline Ceftazidime & Cephalosporin & $\leq 2$ & $\leq 4$ & 8 & $\geq 16$ & $S$ \\
\hline Ceftriaxone & Cephalosporin & $\leq 1$ & $\leq 1$ & 2 & $\geq 4$ & $S$ \\
\hline Gentamicin & Aminoglycoside & 0.25 & $\leq 4$ & 8 & $\geq 16$ & $S$ \\
\hline Imipenem & Carbapenem & 0.5 & $\leq 1$ & 2 & $\geq 4$ & $S$ \\
\hline Levofloxacin & Fluoroquinolone & 0.19 & $\leq 0.5$ & 1 & $\geq 2$ & $S$ \\
\hline Meropenem & Carbapenem & 0.047 & $\leq 1$ & 2 & $\geq 4$ & $\mathrm{~S}$ \\
\hline Nitrofurantoin & Nitrofuran & $>64$ & $\leq 32$ & 64 & $\geq 128$ & 1 \\
\hline Piperacillin-Tazobactam & Penicillin-beta-lactamase inhibitor & $\leq 2 / 4$ & $\leq 16 / 4$ & $32 / 4-64 / 4$ & $\geq 128 / 4$ & $\mathrm{~S}$ \\
\hline Polymyxin B & Polymyxins & 0.5 & $\leq 2$ & 4 & 8 & $\mathrm{~S}$ \\
\hline Tetracycline & Tetracycline & $\leq 2$ & $\leq 4$ & 8 & $\geq 16$ & S \\
\hline Tigecycline & Glycylcycline & 0.38 & $\leq 2$ & 4 & $\geq 8$ & $S$ \\
\hline Tobramycin & Aminoglycoside & 0.5 & $\leq 4$ & 8 & $\geq 16$ & $S$ \\
\hline Trimethoprim-Sulfamethoxazole & Dihydrofolate reductase inhibitor & $0.5 / 9.5$ & $\leq 2 / 38$ & & $\geq 4 / 76$ & $\mathrm{~S}$ \\
\hline
\end{tabular}

Abbreviations: I = intermediate; $\mathrm{MIC}=$ minimum inhibitory concentration; $\mathrm{R}=$ resistant; $\mathrm{S}=$ sensitive.

* Quantitative determination of MIC conducted on Vitek 2 and Phoenix 100 testing systems. Sensitivity was interpreted according to Clinical and Laboratory Standards Institute/Food and Drug Administration guidelines.

\section{References}

1. Kim D, Baik KS, Kim MS, et al. Shewanella haliotis sp. nov., isolated from the gut microflora of abalone, Haliotis discus hannai. Int J Syst Evol Microbiol 2007;57:2926-31. https://doi.org/10.1099/ijs.0.65257-0

2. Zhang F, Fang Y, Pang F, et al. Rare Shewanella spp. associated with pulmonary and bloodstream infections of cancer patients, China: a case report. BMC Infect Dis 2018;18:454. https://doi.org/10.1186/ s12879-018-3354-8

3. Vignier N, Barreau M, Olive C, et al. Human infection with Shewanella putrefaciens and $S$. algae: report of 16 cases in Martinique and review of the literature. Am J Trop Med Hyg 2013;89:151-6. https://doi. org/10.4269/ajtmh.13-0055
4. Janda JM. Shewanella: a marine pathogen as an emerging cause of human disease. Clin Microbiol Newsl 2014;36:25-9. https://doi.org/10.1016/j. clinmicnews.2014.01.006

5. Wu ZY, Liu PY, Tseng SY, Lee YH, Ho SP. Characteristics and phylogeny of Shewanella haliotis isolated from cultivated shellfish in Taiwan. Can J Infect Dis Med Microbiol 2018;2018:9895148. https://doi. org/10.1155/2018/9895148 